\title{
Elevated expression of SATB1 is involved in pancreatic tumorigenesis and is associated with poor patient survival
}

\author{
LEI GUO $^{1 *}$, JIANJIANG ZHENG ${ }^{1 *}$, TAO YU ${ }^{2}$, YUEQUAN LIU $^{1}$ and LUKUN DUO ${ }^{1}$ \\ ${ }^{1}$ Department of Pancreatic Surgery, People's Hospital of Xinjiang, Urumqi, Xinjiang 830000; \\ ${ }^{2}$ Department of Surgery, People's Hospital of Wujiaqu Wujiaqu, Xinjiang 831300, P.R. China
}

Received September 13, 2016; Accepted May 26, 2017

DOI: $10.3892 / \mathrm{mmr} .2017 .7683$

\begin{abstract}
Special AT-rich sequence-binding protein 1(SATB1) is a master chromatin organizer which has been reported to be implicated in tumor progression in breast and lung cancer. However, its functions in pancreatic tumorigenesis have yet to be elucidated. In the present study, the involvement of SATB1 in pancreatic cancer development was investigated in human BxPC-3 pancreatic adenocarcinoma cells. Short hairpin (sh) RNA was used to stably downregulate SATB1 expression, and functional assays, including cell proliferation, colony formation, soft agar and migration assays, were performed in vitro. In addition, a mouse pancreatic cancer xenograft model was created to examine the tumor-promoting properties of SATB1 in vivo. The present findings demonstrated that stable knockdown of SATB1 expression inhibited the proliferation, colony formation, anchorage-independent growth and suppressed the migratory capabilities of BxPC-3 cells in vitro. In addition, SATB1 downregulation significantly inhibited tumor growth in xenografted mice in vivo. Furthermore, SATB1 was revealed to be upregulated in human pancreatic cancer tissue samples compared with matched non-cancerous adjacent tissues, and high SATB1 expression was associated with poor patient survival. Overall, the present study demonstrated that SATB1 promoted the proliferation of pancreatic cancer cells in vitro. In addition, SATB1 expression was revealed to be upregulated in human pancreatic cancer tissues and its upregulation was associated with poor patient survival. Therefore, SATB1 may have potential as a novel prognostic biomarker and therapeutic target for the treatment of patients with pancreatic cancer.
\end{abstract}

Correspondence to: Dr Yuequan Liu, Department of Pancreatic Surgery, People's Hospital of Xinjiang, 152 Longquan Street, Urumqi, Xinjiang 830000, P.R. China

E-mail: liuyuequan1@gmail.com

${ }^{*}$ Contributed equally

Key words: special AT-rich sequence-binding protein 1, pancreatic cancer, tumor progression, poor survival

\section{Introduction}

Pancreatic cancer is one of the most common causes of malignancy-associated morbidities and mortalities worldwide (1). Despite recent developments in surgical treatments and the efficacy of chemotherapeutic agents, the overall prognosis for patients with advanced pancreatic cancer remains poor, whereas the survival rate has not improved in the past few decades. This may be attributed to the asymptomatic nature of pancreatic cancer until the advanced stages of the disease, which constitutes a challenge for early diagnosis (2). The 5-year overall survival rate for patients with advanced pancreatic cancer is $<10 \%$ (1). In China, the 5 -year survival rate in patients with pancreatic cancer is $4.1 \%$ and the median survival time is limited to 3.9 months (3). Therefore, the need to identify novel prognostic biomarkers for the detection of pancreatic cancer at an early stage is of primary concern.

The special AT-rich sequence-binding protein 1 (SATB1) is a nuclear matrix-associated protein which is involved in higher-order chromatin organization and in the regulation of tissue-specific gene expression $(4,5)$. SATB1 is primarily expressed in thymocytes and facilitates thymocyte development through its interaction with the Wnt- $\beta$-catenin signaling pathway $(5,6)$. SATB1 has been associated with the development of several types of cancer, including glioma, colorectal, breast, lung and kidney cancers (7-11). Various genes that are regulated by SATB1 have been implicated in carcinogenesis, including erbB-2, Abelson murine leukemia viral oncogene homolog 1, matrix metalloproteinase 2, E-cadherin, vascular endothelial growth factor $\mathrm{B}$, transforming growth factor- $\beta 1$ and kisspeptin $(12,13)$. In addition, upregulation of SATB1 expression has been associated with unfavorable clinicopathological features and poor patient survival $(8,10,14)$, whereas SATB1 depletion has been reported to suppress the proliferation, growth and invasion of breast cancer cells, through the modulation of gene expression (15). Furthermore, silencing of SATB1 expression has been demonstrated to prevent tumor growth and metastasis, whereas transduction of SATB1 into non-metastatic cells promotes tumor invasion in mice (16). However, the effects of SATB1 on the development and progression of pancreatic cancer have yet to be elucidated.

In the present study, the effects of SATB1 downregulation on pancreatic cancer cell proliferation and tumorigenic properties were investigated in vitro and in vivo. In addition, 
the expression levels of SATB1 in tumor tissue samples from patients with pancreatic cancer were detected and association with patient survival was investigated.

\section{Materials and methods}

Cell culture and treatment. The human BxPC-3 pancreatic adenocarcinoma cell line was purchased from American Type Culture Collection (Manassas, VA, USA). Cells were cultured in Dulbecco's modified Eagle's medium (DMEM; Thermo Fisher Scientific, Inc., Waltham, MA, USA) supplemented with $10 \%$ fetal bovine serum (FBS; Thermo Fisher Scientific, Inc.), and maintained at $37^{\circ} \mathrm{C}$ in a $5 \% \mathrm{CO}_{2}$ atmosphere.

Patient samples. The present study was approved by the Institutional Research Ethics Committee of the People's Hospital of Xinjiang (Urumqi, China). Written informed consent was obtained from patients prior to enrollment in the present study. A total of 48 patients who were admitted in People's Hospital of Xinjiang between 2013 and 2015 were included in the present study. Patient characteristics are presented in Table I. Patients were diagnosed with pancreatic cancer based on histopathological examination. No patients received chemotherapy or radiotherapy prior to surgery. Cancer tissue and adjacent normal tissue (distance $>10 \mathrm{~cm}$ from the primary tumor) samples were isolated from patients who underwent tumor resection, and were immediately frozen in liquid nitrogen and stored at $-80^{\circ} \mathrm{C}$. Tissue samples were fixed in $10 \%$ neutral-buffered formalin at room temperature for $24 \mathrm{~h}$. Following fixation, tissue samples were dehydrated by immersion in increasing concentrations of alcohol. Alcohol was cleared with xylene and tissues were embedded in paraffin by heating to $60^{\circ} \mathrm{C}$ and allowed to harden at room temperature overnight.

Lentiviral transduction and stable colony selection. For the production of the lentivirus, $1 \mu \mathrm{g}$ control short hairpin (sh)RNA (cat no. sc-108060; Santa Cruz Biotechnology, Inc., Dallas, TX, USA) or SATB1-targeting shRNAs (cat no. sc-36460; Santa Cruz Biotechnology, Inc.) were co-transfected with $1 \mu \mathrm{g}$ packaging plasmids $(0.4 \mu \mathrm{g}$ pMD2G and $0.6 \mu \mathrm{g}$ psPAX2; Santa Cruz Biotechnology, Inc.) into human 293FT cells (80\% confluent) in DMEM supplemented with $10 \%$ FBS, using Effectene transfection reagent (Qiagen, Inc., Valencia, CA, USA) according to the manufacturer's protocol. Lentiviral supernatants were collected $48 \mathrm{~h}$ post-transfection and filtered through a $0.45 \mu \mathrm{m}$ filter to remove debris. BxPC-3 cells cultured in DMEM supplemented with $10 \%$ FBS (80\% confluent) were transduced at room temperature with $500 \mu \mathrm{l}$ of viral supernatants at a multiplicity of infection of 10 , containing $4 \mu \mathrm{g} / \mathrm{ml}$ Polybrene transfection reagent (Sigma-Aldrich; Merck KGaA, Darmstadt, Germany) for $18 \mathrm{~h}$, and resistant colonies were selected with $2 \mu \mathrm{g} / \mathrm{ml}$ puromycin (Sigma-Aldrich; Merck $\mathrm{KGaA}$ ) for 7 days. Successful transduction was confirmed by western blotting. Control cells were transduced with control shRNA.

Western blot analysis. Total proteins were extracted from 200,000 BxPC-3 cells following shRNA transduction, using Laemmli SDS reducing buffer $(50 \mathrm{mM}$ Tris- $\mathrm{HCl} \mathrm{pH} 6.8,2 \%$
SDS and $10 \%$ glycerol) at $4{ }^{\circ} \mathrm{C}$, boiled and quantified using a bicinchoninic acid protein assay. Equal amounts $(30 \mu \mathrm{g})$ of extracted protein samples were resolved by $8-10 \%$ PAGE and transferred onto polyvinylidene difluoride membranes. The membranes were blocked with $3 \%$ milk at room temperature for $1 \mathrm{~h}$, incubated with primary antibodies against SATB1 (cat no. ab92307; 1:1,000; Abcam, Cambridge, UK) and GAPDH (cat no. 2118; 1:2,000; Cell Signaling Technology, Inc., Danvers, MA, USA) for $1 \mathrm{~h}$ at room temperature, followed by incubation with horseradish peroxidase-conjugated goat anti-rabbit secondary antibody (cat no. sc-2030, Santa Cruz Biotechnology, Inc.) at a dilution of 1:5,000 at room temperature for $1 \mathrm{~h}$. Protein bands were visualized by enhanced chemiluminescence using the SuperSignal West Pico or Femto Chemiluminescent Substrate kits (Thermo Fisher Scientific, Inc.). Blots were semi-quantified using ImageJ software version 1.41 (National Institutes of Health, Bethesda, MD, USA) (17).

Cell proliferation assay. BxPC-3 cells stably expressing control shRNA or SATB1 shRNA were seeded in DMEM supplemented with $10 \%$ FBS at a density of 10,000 cells/well in 6-well plates in triplicate on day 0. Cells were trypsinized and counted using a TC20 Automated Cell Counter (Bio-Rad Laboratories, Inc., Hercules, CA, USA) on days 0, 1, 2 and 3. Each experiment was performed twice using cells from different suspensions.

Colony formation assay. BxPC-3 cells stably expressing control shRNA or SATB1 shRNA were seeded in 6-well plates at a density of 1,000 cells/well and cultured in DMEM supplemented with $10 \% \mathrm{FBS}$ at $37^{\circ} \mathrm{C}$. Cells were cultured for 1 week and then washed three times with PBS, fixed in $4 \%$ paraformaldehyde for $15 \mathrm{~min}$, and stained with $0.1 \%$ crystal violet for $30 \mathrm{~min}$ at room temperature. Subsequently, the colonies (diameter, $>1 \mathrm{~mm}$ ) were carefully washed with PBS until the background was clear and visualized under an optical microscope. Colony formation efficiency was calculated as the number of colonies divided by 1,000 and normalized to control shRNA infected cells using ImageJ software verson 1.41.

Soft agar assay. BxPC-3 cells (1,000 cells) stably expressing control shRNA or SATB1 shRNA were suspended in $0.375 \%$ Noble agar (Difco; BD Biosciences, Franklin Lakes, NJ, USA) in DMEM supplemented with $10 \%$ FBS and overlaid on $0.75 \%$ Noble agar in 24-well plates. Colonies were allowed to grow for 7-10 days in the growth medium. Colony formation efficiency was calculated according to the following formula: Colony formation efficiency $=($ mean number of colonies/well $) /$ (number of seeded cells/well). Colonies with a diameter $>0.1 \mathrm{~mm}$ were measured and counted, and the mean was used. Data was expressed as fold-change compared to cells expressing the control vector. GraphPad Prism software version 5 (GraphPad Software, Inc., La Jolla, CA, USA) was used for analysis.

Matrigel invasion assay. A total of $10^{5} \mathrm{BxPC}-3$ cells stably expressing control shRNA or SATB1 shRNA were serum-starved overnight, suspended in DMEM and plated into the upper chambers of $8.0-\mu \mathrm{m}$ pore Transwell inserts which were coated with $400 \mu \mathrm{g} / \mathrm{ml}$ Matrigel (BD Biosciences). 
Table I. Characteristics of patients enrolled in the present study.

\begin{tabular}{lc}
\hline Characteristics & Patients \\
\hline Age at diagnosis, median (range) & $59(34-77)$ \\
Age at time of study, median (range) & $61(36-79)$ \\
Gender & \\
Male, n (\%) & $22(45.8)$ \\
Female, n (\%) & $26(54.2)$ \\
Total, n & 48 \\
Pathology & $43(89.5)$ \\
Ductal adenocarcinoma, n (\%) \\
Adenocarcinoma associated with \\
intraductal papillary mucinous \\
neoplasm, n (\%)
\end{tabular}

A total of $500 \mu \mathrm{l}$ medium supplemented with $10 \%$ FBS was added to the lower chambers as a chemoattractant. Cells were incubated at $37^{\circ} \mathrm{C}$ for $24 \mathrm{~h}$. Non-migrated cells on the top of the membrane were removed using cotton swabs, and cells that had migrated to the lower membrane were stained with $6 \%$ glutaraldehyde $/ 0.5 \%$ crystal violet solution at room temperature for $30 \mathrm{~min}$. Experiments were performed in triplicate. Invaded cells were visualized under an optical microscope, and counted using ImageJ software (17), by averaging the number of stained cells/field of view in 5 random fields/chamber.

IHC. Paraffin-embedded tumor and adjacent normal 5- $\mu \mathrm{m}$ thick tissue sections were subjected to antigen retrieval by heating in a microwave at $100^{\circ} \mathrm{C}$ for $10 \mathrm{~min}$ in $0.1 \mathrm{M}$ citric acid buffer ( $\mathrm{pH}$ 6.0), deparaffinized in xylene and rehydrated in graded ethanol. Endogenous peroxidase activity was blocked with $3 \%$ hydrogen peroxide for $1 \mathrm{~h}$ at room temperature, following permeabilization with ice-cold $100 \%$ methanol for $10 \mathrm{~min}$ at $-20^{\circ} \mathrm{C}$ and rinsed in PBS for $5 \mathrm{~min}$. Sections were then incubated with an anti-SATB1 antibody (1:200; cat no. ab92307; Abcam) at $4^{\circ} \mathrm{C}$ overnight. Following incubation with HRP-conjugated secondary antibodies (cat no. BA-1000; 1:200; Vector Laboratories, Inc., Burlingame, CA, USA) at room temperature for $1 \mathrm{~h}$, the slides were developed in $0.05 \%$ 3,3'-diaminobenzidine (Vector Laboratories, Inc.) containing $0.01 \%$ hydrogen peroxide at room temperature for $1 \mathrm{~min}$. As a negative control, sections were incubated with normal goat serum (Invitrogen; Thermo Fisher Scientific, Inc.), instead of primary antibodies, at $4^{\circ} \mathrm{C}$ overnight. The density of the staining was ranked as follows: 0 , no staining; 1 , mild staining; 2 , moderate staining; and 3 , intense staining. The extent of staining was scored as follows: 0 , no positive cells; 1 , positive cells cover $<10 \%$ of total area; 2 , positive cells cover $10-50 \%$ of total area; and 3, positive cells cover $>50 \%$ of total area. The final staining score was obtained by multiplying the intensity score with the extent score. The samples were classified into 2 groups according to the final score: Low (0-4) and high (5-9).

Mouse xenografts. Animal experiments were approved by the Institutional Animal Care and Use Committee of the National
Cancer Center (Urumqi, China). BXPC-3 cells expressing control shRNA or SATB1 shRNA $\left(3 \times 10^{6}\right.$ cells/injection) were subcutaneously injected into both flanks of 10 female nude mice (age, 6 weeks; weight, $\sim 25 \mathrm{~g}$ ). The mice were purchased from the Chinese University of Hong Kong (Hong Kong, China) and maintained in individually ventilated cages under a 12-h light/dark cycle at $20-22^{\circ} \mathrm{C}$ and $40-60 \%$ relative humidity with free access to food and water. Between days 8 to 26 post-implantation, tumor volumes were measured using a caliper according to the following formula: Tumor volume $\left(\mathrm{mm}^{3}\right)=$ tumor length $\mathrm{x}$ (tumor width) $\left.)^{2} / 2\right]$. Data were expressed as the mean tumor volume \pm standard deviation. Mice were sacrificed 26 days post-implantation by $\mathrm{CO}_{2}$ inhalation.

RNA isolation and reverse transcription-quantitative polymerase chain reaction ( $R T-q P C R)$. Total RNA was extracted from cancer tissue and paired normal mucosal tissue samples isolated from 48 patients with pancreatic cancer using TRIzol ${ }^{\circledR}$ (Invitrogen; Thermo Fisher Scientific, Inc.), according to the manufacturer's protocol. Total RNA $(1 \mu \mathrm{g})$ was reverse transcribed into cDNA using RevertAid ${ }^{\mathrm{TM}}$ First Strand cDNA Synthesis kit (Thermo Fisher Scientific, Inc.). The reaction volume was $20 \mu \mathrm{l}$ and contained $1 \mu \mathrm{g}$ RNA and $1 \mu \mathrm{ldT}$ primer, $4 \mu \mathrm{l}$ reaction buffer, $2 \mu \mathrm{l} \mathrm{dNTP}, 0.5 \mu \mathrm{l}$ inhibitor and $0.5 \mu \mathrm{l}$ reverse transcriptase. The temperature protocol was as follows: At $65^{\circ} \mathrm{C}$ for $10 \mathrm{~min}$, at $25^{\circ} \mathrm{C}$ for $10 \mathrm{~min}$, at $55^{\circ} \mathrm{C}$ for $30 \mathrm{~min}$, and then at $85^{\circ} \mathrm{C}$ for $5 \mathrm{~min}$. PCR was performed on cDNA using a ViiA ${ }^{\mathrm{TM}} 7$ Real-Time PCR system (Applied Biosystems; Thermo Fisher Scientific, Inc.) with SYBR ${ }^{\circledR}$ Premix DimerEraser ${ }^{\mathrm{TM}}$ (Takara Bio, Inc., Otsu, Japan) according to the manufacturer's protocol. Each experiment was performed in triplicate. Primer sequences were as follows: SABT1, forward 5'-AAAAGAAATCGGACCACCAAGC-3', reverse 5'-TGTGGTACGGAGCTGATCG-3'; and GAPDH, forward 5'-GGCCAAGGTCATCCATGACAA-3' and reverse 5'-TCTTCTGACACCTACCGGGGA-3'. Thermocycling conditions were as follows: Initial denaturation at $95^{\circ} \mathrm{C}$ for 2 min, followed by 40 cycles at $95^{\circ} \mathrm{C}$ for $10 \mathrm{sec}$, and at $60^{\circ} \mathrm{C}$ for $30 \mathrm{sec}$, with an extension at $72^{\circ} \mathrm{C}$ for $30 \mathrm{sec}$. The specificity of the amplification products was confirmed by the exhibition of a singlet in the melting curve and gene expression was quantified according to the comparative $\mathrm{Cq}$ method (18).

Statistical analysis. Data are expressed as the mean \pm standard deviation of 3 independent experiments. Data were analyzed using one-way analysis of variance (ANOVA) or mixed-factorial ANOVA, where appropriate. Multiple comparisons were then further investigated using Tukey post hoc test. $\mathrm{P}<0.05$ was considered to indicate a statistically significant difference. Survival curves were constructed using the Kaplan-Meier method and compared using log-rank test. Statistical analyses were performed using SPSS software version 16 (SPSS, Inc., Chicago, IL, USA) and GraphPad Prism software version 5.0 (GraphPad Software, Inc., La Jolla, CA, USA).

\section{Results}

SATB1 knockdown inhibits the proliferation and suppresses the clonogenicity of pancreatic cancer cells. To examine the functions of SATB1 on pancreatic cancer cell growth, stable 
A

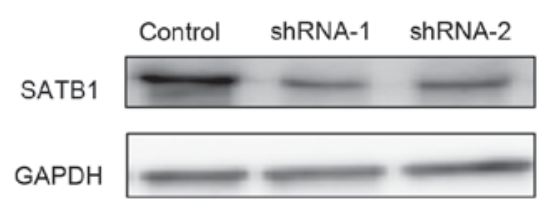

B

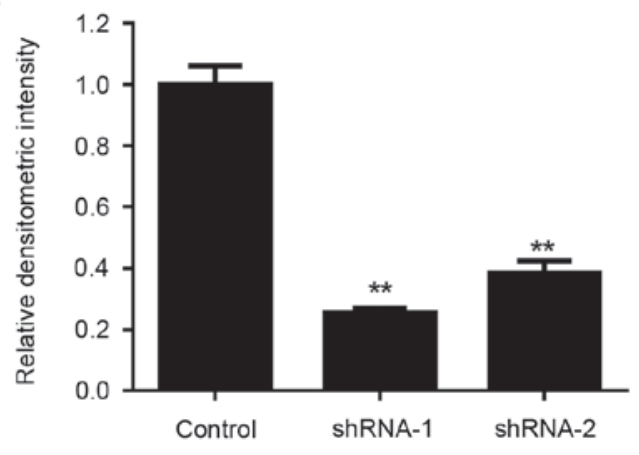

Figure 1. Downregulation of SATB1 expression using shRNAs in human BxPC-3 pancreatic adenocarcinoma cells. (A) Silencing of SATB1 expression in BxPC-3 cells using 2 independent shRNAs targeting SATB1 was confirmed using western blot analysis. (B) Blots were semi-quantified using densitometry. Control cells were transduced with control shRNA. Data are expressed as the mean \pm standard deviation. ${ }^{* *} \mathrm{P}<0.01$ vs. Control. SATB, special AT-rich sequence-binding protein; sh, short hairpin.

SATB1-knockdown BxPC-3 cells were established using RNA interference. As presented in Fig. 1, following transduction with 2 independent shRNAs targeting SATB1, the protein expression levels of SATB1 in BxPC-3 cells were significantly downregulated. Knockdown of SATB1 expression significantly inhibited the proliferation of BxPC-3 cells compared with control cells, as demonstrated by a cellular proliferation assay (Fig. 2A). In addition, BxPC-3 cells transduced with SATB1-specific shRNAs exhibited significantly suppressed colony formation capabilities (Fig. 2B), thus suggesting that SATB1 may enhance the proliferation and clonogenicity of pancreatic cancer cells.

SATB1 knockdown suppresses the anchorage-independent growth and invasion of pancreatic cancer cells. Soft agar growth and Matrigel invasion assays were performed to evaluate the effects of SATB1 knockdown on the tumorigenic properties of pancreatic cancer cells. As presented in Fig. 3, following the downregulation of SATB1 expression, the anchorage-independent growth of BxPC-3 cells was significantly inhibited. In addition, the invasive capabilities of cancer cells were significantly reduced following SATB1 shRNA transduction, as demonstrated by the Matrigel invasion assay.

SATB1 knockdown inhibits tumor growth in a pancreatic cancer xenograft mouse model. To investigate the effects of SATB1 on pancreatic cancer cell growth in vivo, a mouse xenograft model was established following the subcutaneous injection of BxPC-3 cells expressing control shRNA or SATB1 shRNA-1 into nude mice. Tumor growth was monitored for 26 days post-xenotransplantation. As demonstrated in Fig. 4 , SATB1 downregulation significantly reduced the weight and growth rate of tumor xenografts in vivo. These findings suggested that SATB1 may serve a role in promoting tumor growth in vivo.

SATB1 expression is upregulated in pancreatic cancer tissues and is associated with poor prognosis. Pancreatic cancer tissue and matched non-cancerous adjacent tissue samples were isolated from 48 patients with pancreatic cancer.
Semi-quantitative RT-PCR demonstrated that the mRNA expression levels of SATB1 were significantly upregulated in pancreatic cancer tissues compared with in matched control samples (Fig. 5A). In addition, IHC results revealed that pancreatic cancer tissues exhibited stronger SATB1 staining compared with non-cancerous tissue samples (Fig. 5B). The patient cohort was divided into low and high SATB1 expression groups according to the IHC scoring, and a Kaplan-Meier survival analysis was performed. As presented in Fig. 5C, patients with high SATB1 expression had significantly shorter overall survival times compared with patients with low SATB1 expression scores.

\section{Discussion}

Pancreatic cancer is one of the most malignant types of cancer, and is characterized by a high incidence of metastasis and a low 5-year survival rate. Platinum-based antineoplastic drugs or paclitaxel combination chemotherapy are the standard regimens used for the treatment of pancreatic cancer; however, the recurrence rate of the disease remains as high as $85 \%$ (2) Therefore, the need to identify novel therapeutic targets for the treatment of pancreatic cancer is of primary concern.

SATB1 has been suggested to regulate gene expression by selectively tethering far-distal specialized DNA sequences to its cage-like network, and scaffolding them with chromatin-modifying and transcription factors in order to accurately modulate gene expression $(19,20)$. Previous studies have reported that SATB1 is overexpressed in metastatic breast cancer cell lines and in tissue specimens isolated from patients with metastatic breast carcinoma $(21,22)$. Depletion of SATB1 has been demonstrated to suppress cancer cell growth and inhibit tumor metastasis, whereas its overexpression promotes tumor growth and lung colonization in breast cancer (15). A previous study reported that transient silencing of SATB1 expression inhibits the proliferation and invasion of small cell lung cancer cells (23). However, the effects of SATB1 downregulation on pancreatic cancer tumorigenesis have yet to be elucidated. 
A

BxPC-3

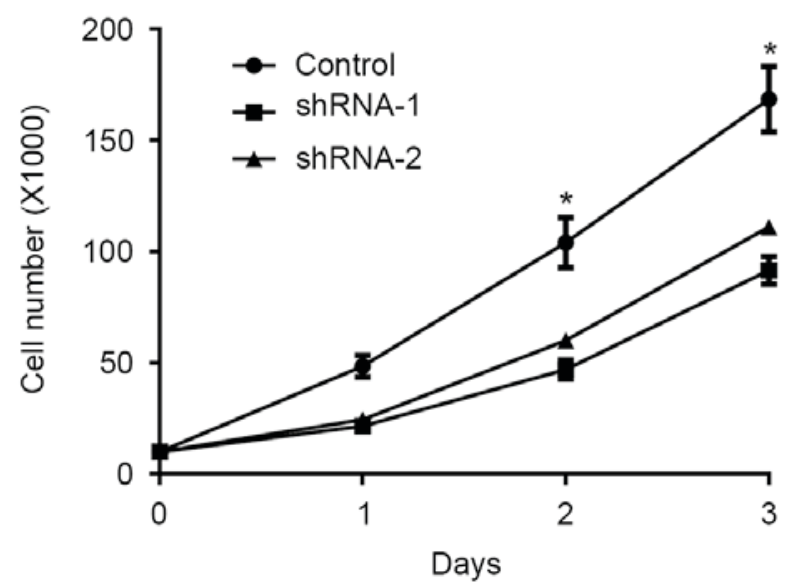

B
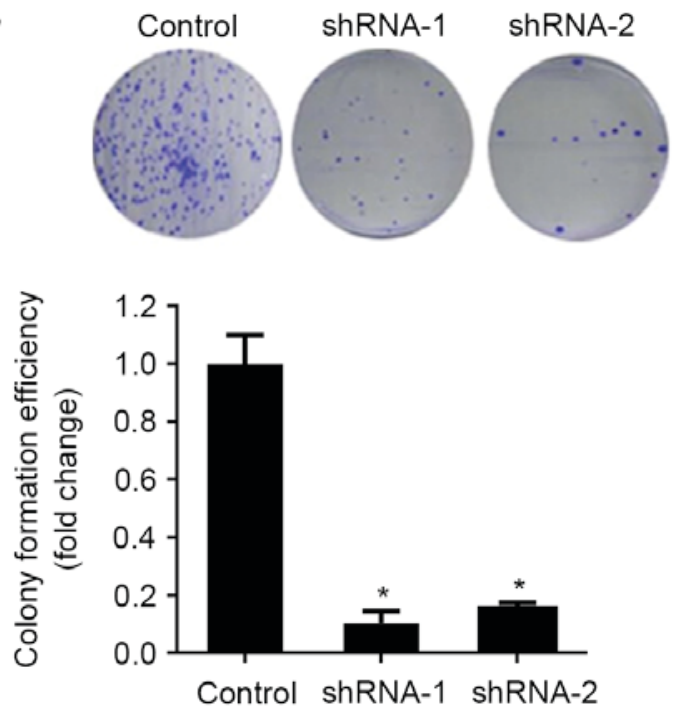

Figure 2. Downregulation of SATB1 expression suppresses the proliferation and clonogenicity of human BxPC-3 pancreatic adenocarcinoma cells. (A) Growth curves of BxPC-3 cells stably expressing control or SATB1-targeting shRNAs. (B) Colony formation capabilities of BxPC-3 cells stably expressing control or SATB1-targeting shRNAs. Data are expressed as the mean \pm standard deviation. ${ }^{*}<<0.05$ vs. Control. SATB, special AT-rich sequence-binding protein; sh, short hairpin.

A

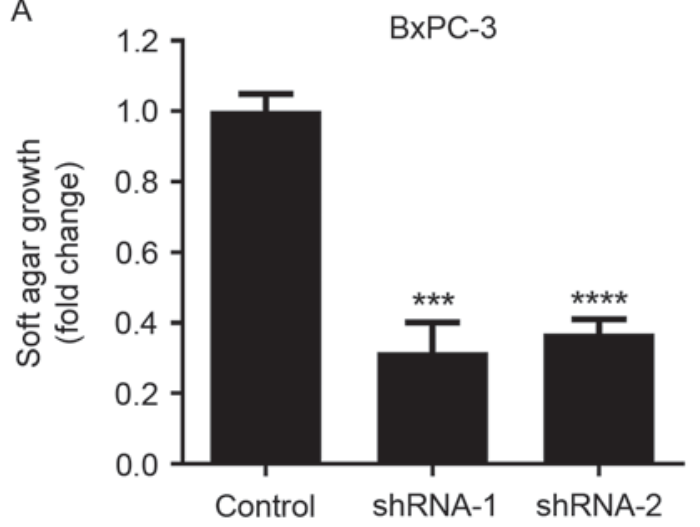

B

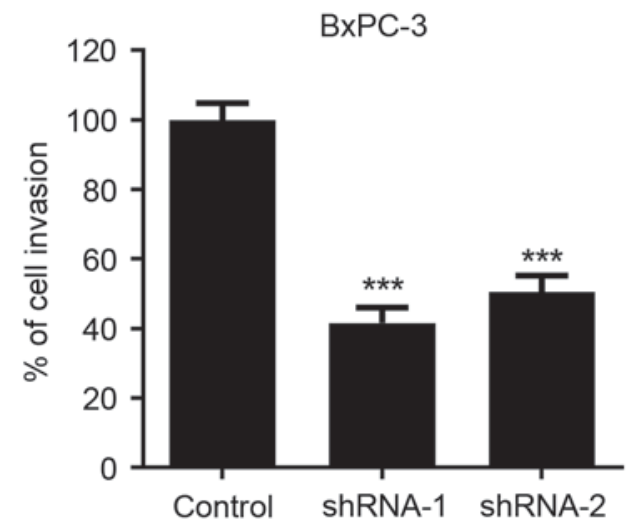

Figure 3. Downregulation of SATB1 expression suppresses the anchorage-independent growth and invasion of human BxPC-3 pancreatic adenocarcinoma cells. (A) A soft agar growth assay detected the anchorage-independent growth of BxPC-3 cells stably expressing control or SATB1-targeting shRNAs. (B) A Matrigel invasion assay assessed the invasive capabilities of BxPC-3 cells stably expressing control or SATB1-targeting shRNAs. Data are expressed as the mean \pm standard deviation. ${ }^{\cdots} \mathrm{P}<0.001,{ }^{* * * * *} \mathrm{P}<0.0001$ vs. Control. SATB, special AT-rich sequence-binding protein; sh, short hairpin.

A

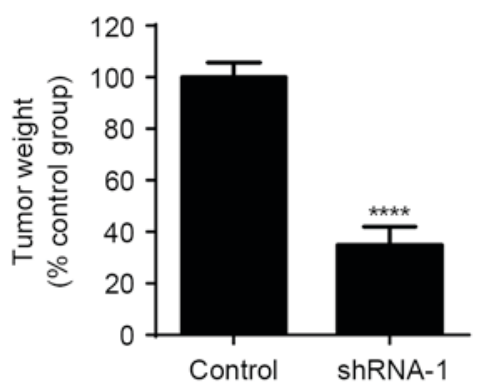

B

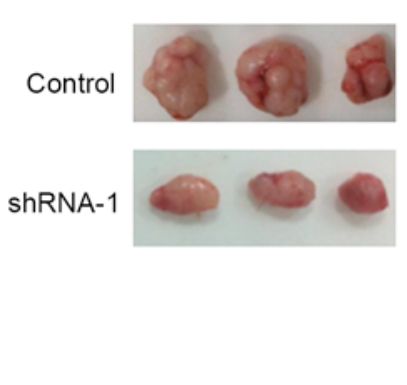

\section{BXPC-3}

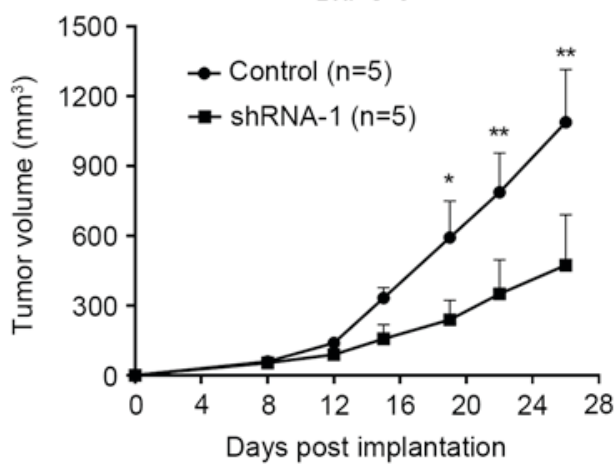

Figure 4. Downregulation of SATB1 expression inhibits tumor growth in a mouse xenograft model. (A) Weight and representative images of the xenograft tumors isolated from mice implanted with human BxPC-3 pancreatic adenocarcinoma cells stably expressing control or SATB1-targeting shRNA-1. (B) Growth curves of tumor xenografts isolated from mice implanted with human BxPC-3 pancreatic adenocarcinoma cells stably expressing control or SATB1-targeting shRNA-1 ( $\mathrm{n}=5$ mice/group). Data are expressed as the mean \pm standard deviation. ${ }^{*} \mathrm{P}<0.05,{ }^{* *} \mathrm{P}<0.01,{ }^{* * * * *} \mathrm{P}<0.0001$ vs. Control. SATB, special AT-rich sequence-binding protein; sh, short hairpin. 
A

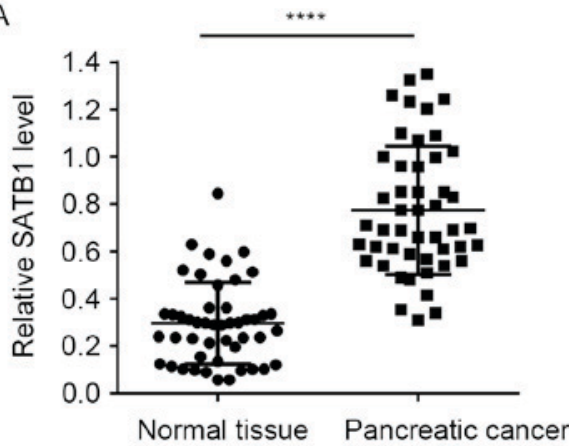

B

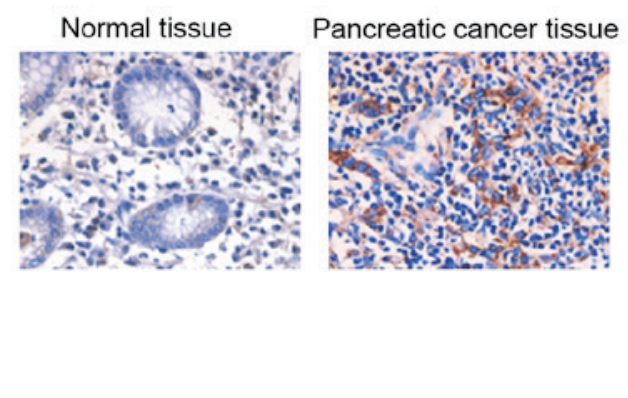

C

Overall survival

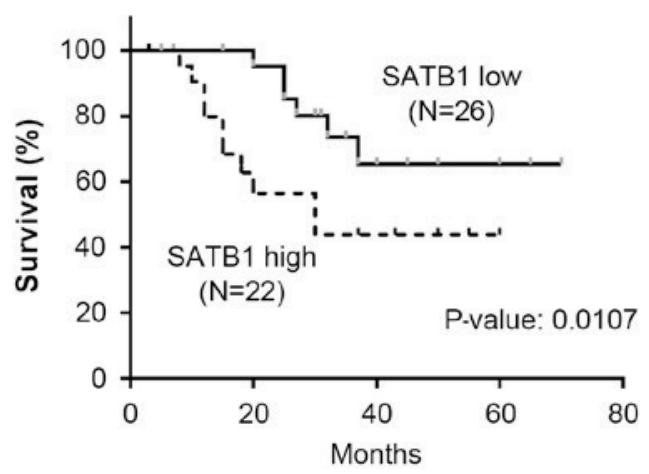

Figure 5. SATB1 expression is upregulated in tumor tissue samples isolated from patients with pancreatic cancer, and is associated with poor survival. (A) SATB1 mRNA expression was assessed in tumor and adjacent non-cancerous tissue samples isolated from 48 patients with pancreatic cancer. Dots represent the mRNA expression level of SATB1 for each patient. (B) Representative immunohistochemical staining of pancreatic cancer and adjacent non-cancerous tissue samples. Magnification, x200. (C) Overall survival in patients with pancreatic cancer with low or high levels of SATB1 expression. Survival curves were constructed using Kaplan-Mier analysis and statistical analysis was performed using the log-rank test. ${ }^{* * * * *} \mathrm{P}<0.0001$. SATB, special AT-rich sequence-binding protein.

In the present study, stable downregulation of SATB1 expression using RNA interference significantly inhibited the proliferative, colony formation and invasive capabilities of BxPC-3 cells, and suppressed soft agar growth. In addition, SATB1 knockdown repressed tumor growth in a xenograft mouse model. SATB1 may be implicated in pancreatic tumorigenesis through the regulation of several genes known to be involved in carcinogenesis; however, further studies are required to investigate the molecular mechanisms and downstream effectors of SATB1 that are involved in the development of pancreatic cancer. Pan et al (24) reported that high SATB1 expression is significantly correlated with the progression and metastasis of breast cancer, and thus with poor disease prognosis. Similarly, in the present study, SATB1 expression was revealed to be significantly upregulated in pancreatic cancer tissues compared with in matched non-cancerous adjacent tissues. Notably, Kaplan-Meier survival analysis revealed that high SATB1 expression was significantly associated with decreased patient survival, thus suggesting that SATB1 may have potential as a prognostic biomarker for patients with pancreatic cancer.

In conclusion, the results of the present study suggested that SATB1 may be implicated in pancreatic tumorigenesis. SATB1 was revealed to be significantly upregulated in pancreatic cancer tissues and was associated with poor survival of patients with pancreatic cancer. Therefore, SATB1 may have potential as a novel therapeutic target for the treatment of patients with pancreatic cancer, and as a biomarker for disease prognosis. However, further studies are required to fully elucidate the molecular mechanisms that underlie the implication of SATB1 in promoting pancreatic cancer progression.

\section{Acknowledgements}

The present study was supported by the People's Hospital of Xinjiang, Urumqi, China (grant no. 829020801).

\section{References}

1. Siegel RL, Miller KD and Jemal A: Cancer statistics, 2016. CA Cancer J Clin 66: 7-30, 2016.

2. Kaur S, Baine MJ, Jain M, Sasson AR and Batra SK: Early diagnosis of pancreatic cancer: Challenges and new developments. Biomark Med 6: 597-612, 2012.

3. Luo J, Xiao L, Wu C, Zheng Y and Zhao N: The incidence and survival rate of population-based pancreatic cancer patients: Shanghai cancer registry 2004-2009. PLoS One 8: e76052, 2013.

4. Nakayama Y, Mian IS, Kohwi-Shigematsu T and Ogawa T: A nuclear targeting determinant for SATB1, a genome organizer in the T cell lineage. Cell Cycle 4: 1099-1106, 2005.

5. Cai S, Han HJ and Kohwi-Shigematsu T: Tissue-specific nuclear architecture and gene expression regulated by SATB1. Nat Genet 34: 42-51, 2003 
6. Notani D, Gottimukkala KP, Jayani RS, Limaye AS, Damle MV, Mehta S, Purbey PK, Joseph J and Galande S: Global regulator SATB1 recruits beta-catenin and regulates $\mathrm{T}(\mathrm{H}) 2$ differentiation in Wnt-dependent manner. PLoS Biol 8: e1000296, 2010.

7. Nodin B, Johannesson H, Wangefjord S, O'Connor DP, Lindquist KE, Uhlén M, Jirström K and Eberhard J: Molecular correlates and prognostic significance of SATB1 expression in colorectal cancer. Diagn Pathol 7: 115, 2012.

8. Zheng J: Is SATB1 a master regulator in breast cancer growth and metastasis? Womens Health (Lond) 4: 329-332, 2008

9. Selinger CI, Cooper WA, Al-Sohaily S, Mladenova DN, Pangon L, Kennedy CW, McCaughan BC, Stirzaker C and Kohonen-Corish MR: Loss of special AT-rich binding protein 1 expression is a marker of poor survival in lung cancer. J Thorac Oncol 6: 1179-1189, 2011.

10. Chu SH, Ma YB, Feng DF, Zhang H, Zhu ZA, Li ZQ and Jiang PC: Upregulation of SATB1 is associated with the development and progression of glioma. J Transl Med 10: 149, 2012.

11. Cheng C, Wan F, Liu L, Zeng F, Xing S, Wu X, Chen X and Zhu Z: Overexpression of SATB1 is associated with biologic behavior in human renal cell carcinoma. PLoS One 9: e97406, 2014.

12. Kohwi-Shigematsu T, Poterlowicz K, Ordinario E, Han HJ Botchkarev VA and Kohwi Y: Genome organizing function of SATB1 in tumor progression. Semin Cancer Biol 23: 72-79, 2013.

13. Mir R, Pradhan SJ and Galande S: Chromatin organizer SATB as a novel molecular target for cancer therapy. Semin Cancer Biol 13: 1603-1615, 2012.

14. Deng YF, Zhou DN, Pan ZY and Yin P: Aberrant SATB1 expression is associated with Epstein-Barr virus infection, metastasis and survival in human nasopharyngeal cells and endemic nasopharyngeal carcinoma. Int J Clin Exp Pathol 7: 2454-2461, 2014.

15. Han HJ, Russo J, Kohwi Y and Kohwi-Shigematsu T: SATB1 reprogrammes gene expression to promote breast tumour growth and metastasis. Nature 452: 187-193, 2008.
16. Neznanov N, Kohwi-Shigematsu T and Oshima RG: Contrasting effects of the SATB1 core nuclear matrix attachment region and flanking sequences of the keratin 18 gene in transgenic mice. Mol Biol Cell 7: 541-552, 1996.

17. Schneider CA, Rasband WS and Eliceiri KW: NIH Image to ImageJ: 25 years of image analysis. Nat Methods 9: 671-675, 2012

18. Livak KJ and Schmittgen TD: Analysis of relative gene expression data using real-time quantitative PCR and the 2(-Delta Delta C(T)) method. Methods 25: 402-408, 2001.

19. Cai S, Lee CC and Kohwi-Shigematsu T: SATB1 packages densely looped, transcriptionally active chromatin for coordinated expression of cytokine genes. Nat Genet 38: 1278-1288, 2006.

20. Yamaguchi H, Tateno $M$ and Yamasaki K: Solution structure and DNA-binding mode of the matrix attachment region-binding domain of the transcription factor SATB1 that regulates the T-cell maturation. J Biol Chem 281: 5319-5327, 2006.

21. Hanker LC, Karn T, Mavrova-Risteska L, Ruckhäberle E, Gaetje R, Holtrich U, Kaufmann M, Rody A and Wiegratz I: SATB1 gene expression and breast cancer prognosis. Breast 20: 309-313, 2011.

22. Patani N, Jiang W, Mansel R, Newbold R and Mokbel K: The mRNA expression of SATB1 and SATB2 in human breast cancer. Cancer Cell Int 9: 18, 2009.

23. Huang B, Zhou H, Wang X and Liu Z: Silencing SATB1 with siRNA inhibits the proliferation and invasion of small cell lung cancer cells. Cancer Cell Int 13: 8, 2013.

24. Pan Z, Jing W, He K, Zhang L and Long X: SATB1 is correlated with progression and metastasis of breast cancers: A meta-analysis. Cell Physiol Biochem 38: 1975-1983, 2016. 